Supplementary information

\title{
A Room Temperature, Solution Phase Method for the Synthesis of Mesoporous Metal Chalcogenide Nanocrystal Based Thin Films with Precisely Controlled Grain Sizes
}

Justin C. Ondry ${ }^{\dagger, \#}$, Shauna Robbennolt ${ }^{\dagger}$, Hyeyeon $\mathrm{Kang}^{\dagger}$, Yan Yan ${ }^{\dagger}$, and Sarah H. Tolbert ${ }^{\dagger, \ddagger}$.

'Department of Chemistry and Biochemistry, UCLA, Los Angeles, California 90095-1569, United States, ${ }^{\ddagger}$ Department of Materials Science \& Engineering and the California NanoSystems Institute, UCLA, Los Angeles, California 90095, United States.

*E-mail: tolbert@chem.ucla.edu

" Present address: Department of Chemistry, University of California at Berkeley, Berkeley, CA 94720

This file includes:

Figure S1: XPS characterization of the mesoporous materials

Figure S2 and S3: additional HRTEM images of PbS/CdS core shell particles 

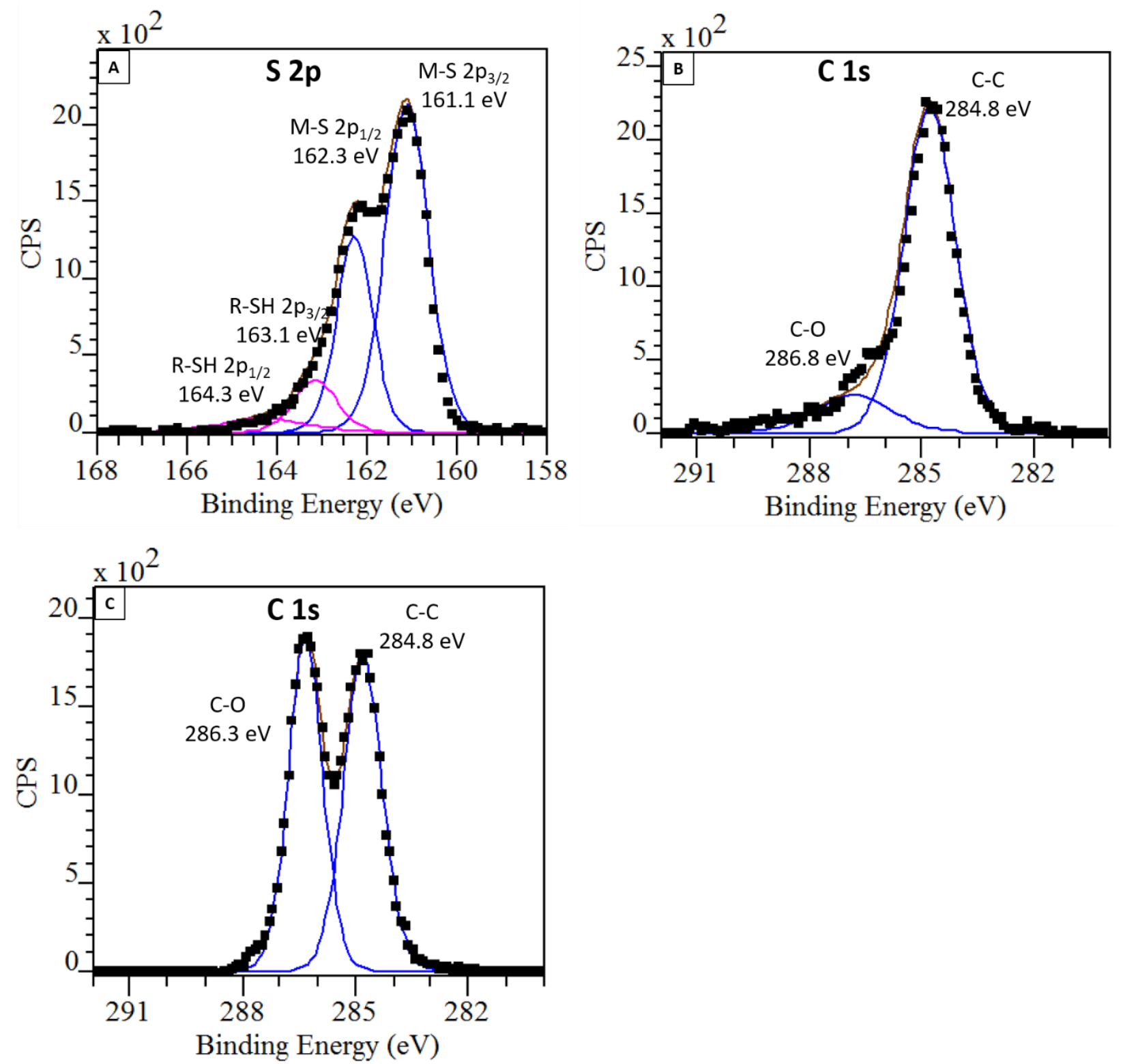

Figure S1: (A) Sulfur 2p XPS from a mesoporous film of EDT cross linked PbS/CdS core shell nanocrystals showing the presence of sulfur signal arising from the metal sulfide nanocrystals and the bound thiolate arising from the EDT bound to the surface. (B) Carbon is XPS from a mesoporous film of EDT cross linked PbS/CdS core shell nanocrystals showing minimal signal from $\mathrm{C}-\mathrm{O}$, which comes from the ethers present in the PEO-b-PBO polymer template. The data thus indicates removal of the majority of the polymer template. (C) $C$ is XPS from pure PEO-b-PBO showing a large C-O signal, as expected from a poly-ether based polymer, along with significant $\mathrm{C}-\mathrm{C}$ signal. We used this data to quantified the wt\% polymer remaining in a mesoporous sample by comparing the integrated area of the $\mathrm{C}-\mathrm{O}$ carbon peak to the integrated area of the metal sulfur peak to determine a ratio of the two elements. We then converted the atom percent $\mathrm{C}-\mathrm{O}$ carbon to a weight using the number of $\mathrm{C}-\mathrm{O}$ carbons in the average PEO-b-PBO and the molecular weight of the polymer. The atom percent sulfur was converted to a mass using the molar mass of the metal sulfide nanocrystals (in this sample, the nanocrystals were $\mathrm{PbS} / \mathrm{CdS}$ core shell nanocrystals and the molar mass used was the weighted average of the two materials based on the core and shell volumes). Based on this analysis, we determine that our samples contain only o.1 wt\% polymer remaining in the sample. Sample initially contained $\sim 30 \%$ polymer based on the synthesis parameters used. 


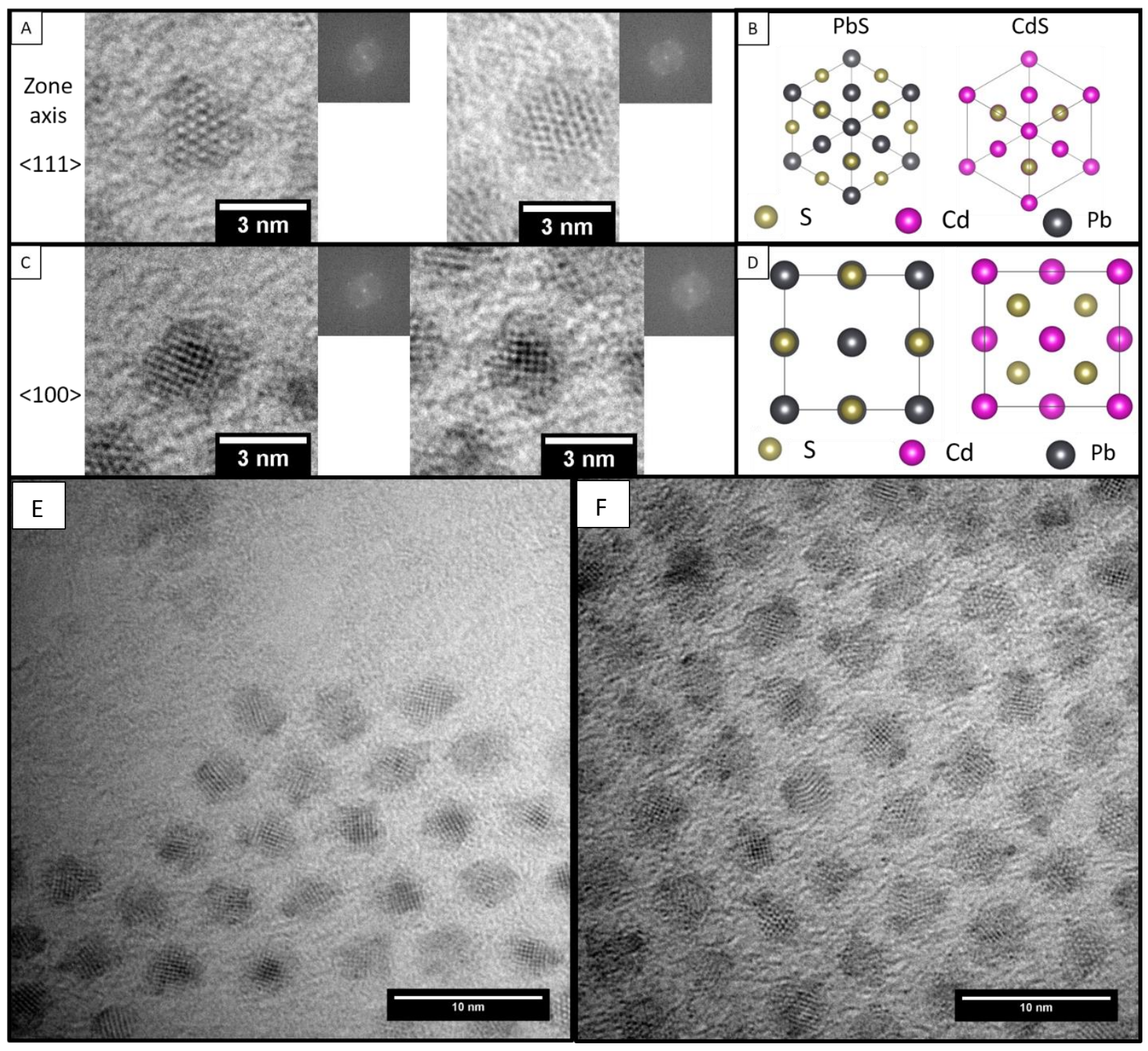

Figure S2: (A) HRTEM images of $\mathrm{PbS} / \mathrm{CdS}$ particles viewed down the <111> zone axis. (B) Visulization of the $\mathrm{PbS}$ and CdS lattices when viewed down the <111> zone axis. (C) HRTEM Images of PbS/CdS particles viewed down the $<100>$ zone axis. (D) Visulization of the $\mathrm{PbS}$ and $\mathrm{CdS}$ lattices when viewed down the $<100>$ zone axis. (E) and (F) wide field HRTEM images of several PbS/CdS nanocrystals showing the diversity of structures that are observed. 


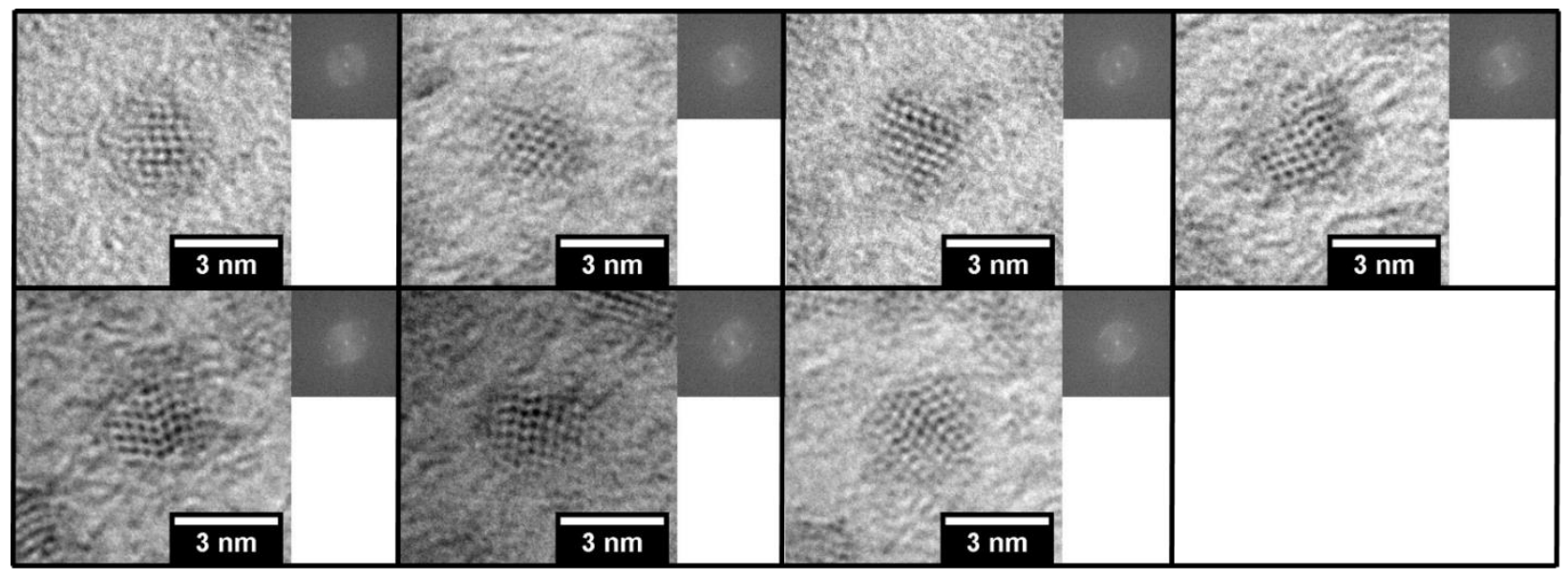

Figure S3: HRTEM images of PbS/CdS core shell particles that contain stacking faults and other defects. 